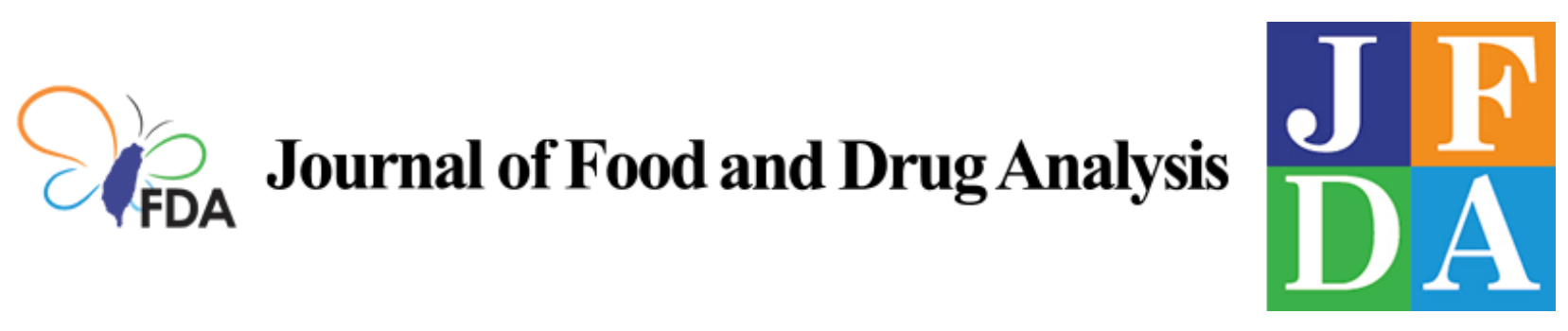

Volume 29 | Issue 3

Article 3

2021

\title{
Comprehensive detection of 120 additives in food using nontargeted MS data acquisition
}

Follow this and additional works at: https://www.jfda-online.com/journal

Part of the Food Science Commons, Medicinal Chemistry and Pharmaceutics Commons, Pharmacology Commons, and the Toxicology Commons

(c) (i) (9)

This work is licensed under a Creative Commons Attribution-Noncommercial-No Derivative Works 4.0 License.

\section{Recommended Citation}

Hsu, Che-Lun; Fang, Mingchih; Tseng, Hui-Chun; Lin, Ya-Tze; Tseng, Su-Hsiang; Wang, Der-Yuan; and Hwang, Deng-Fwu (2021) "Comprehensive detection of 120 additives in food using nontargeted MS data acquisition," Journal of Food and Drug Analysis: Vol. 29 : Iss. 3 , Article 3.

Available at: https://doi.org/10.38212/2224-6614.3366

This Original Article is brought to you for free and open access by Journal of Food and Drug Analysis. It has been accepted for inclusion in Journal of Food and Drug Analysis by an authorized editor of Journal of Food and Drug Analysis. 


\title{
Comprehensive detection of $\mathbf{1 2 0}$ additives in food using nontargeted MS data acquisition
}

\author{
Che-Lun Hsu ${ }^{a, b}$, Mingchih Fang ${ }^{a, *}$, Hui-Chun Tseng ${ }^{b}$, Ya-Tze Lin ${ }^{b}$, Su-Hsiang Tseng ${ }^{b}$, \\ Der-Yuan Wang ${ }^{b}$, Deng-Fwu Hwang ${ }^{a}$ \\ a Department of Food Science, National Taiwan Ocean University, 2 Beining Road, Keelung City 20224, Taiwan
b Division of Research and Analysis, Taiwan Food and Drug Administration, 161-2 Kunyang St., Nangang Dist., Taipei City 11561,
Taiwan
}

Abstract

The compliance assessment on the labeling of food additives is a hard job, because there are nearly thousand legal food additives can be used in food, and countless illegal additives must also deal with. This study developed a nontargeted data acquisition screening method based on liquid chromatography high resolution mass spectrometry (HRMS) in which a precursor ion and two product ions of each analyte are able to be recorded. The high throughput screening method worked as foodomics that characterized and identified every food components as long as they were ionized in terms of theory. The data acquisition method called data independent acquisition (DIA) was achieved by a full scan form $m / z$ 70-1050, and then followed wide window fragmentations of product ions recording. A full scan and the followed fragmentations generated 21 spectra in $2.6 \mathrm{~s}$ contributed about 6 data points for a typical $0.2-0.3$ min width peak in HPLC. A detection database list of 120 additives included 79 colorants, 13 sweeteners, 12 preservatives and 7 antioxidants was established. Thirty-three commercial samples including beverages, candies, and sauces were surveyed for testing additives. Sweeteners (rebaudioside A) and flavoring agents (malic acid and fumaric acid) were found the most under declared additives. HPLC column often do not provide adequate retention for highly polar compounds such as organic acids (flavoring agents). In this study they were coeluted, but were able to be separated and determined by HRMS worked as the secondary separation tool. The surveillance results showed there is still room for food manufacturers to improve the connection between their product information and consumers.

Keywords: Additives, DIA, Fast screening, HRMS, Nontargeted

\section{Introduction}

$\mathrm{F}$ ood additives are required to be labeled on the package of food. Most direct additives belong to parts of the ingredient labels of food products, and some trace amounts of indirect additives are not required to be labeled. Additives used in food commonly include colorants, sweeteners, preservatives, antioxidants, and flavoring agents. These natural and/or artificial additives need to be regulated and monitored before addition, treating, or processing. Food additives are evaluated by authorities such as European Food Safety Authority's (EFSA) or Food and Drug Administration (FDA) to be represented as legal additives, while others not listed in food additive status list are recognized as illegal. The regulations of nations applicable to food additives are vary that force exporters to adjust their product compositions to the importers' markets. Failure to comply may result in adulteration, misbranding, non-compliance and rejection [1]. Therefore, the monitoring and detection of additives in foods has to include both legal and illegal additives depend on their legal states. Besides, some additives such as Sudan dyes, dimethyl yellow and ethoxyquin are illegal or not permitted to use in food for human consumption,

Received 18 March 2021; revised 21 May 2021; accepted 31 May 2021.

Available online 15 September 2021.

* Corresponding author at: National Taiwan Ocean University, Keelung City, Taiwan.

E-mail address: mcfang@mail.ntou.edu.tw (M. Fang). 
but may be found in contaminated foods [2-4]. It is necessary to develop a simultaneous detection method for broadly screening of additives in foods. However, additives with various functions to foods present differences in their physicochemical properties, these make the monitoring of a large group of additives difficult in every step for a method development such as sample preparation, separation and detection.

Various analytical methods reported the detections of a single class of additives. Sagdic [5] detected 18 phenolic compounds including antioxidants by high performance liquid chromatography (HPLC) in grape. Two natural antioxidants were monitored in enriched virgin olive oil trough capillary electrophoresis method [6]. Seven preservative were simultaneously determined in cosmetics by dispersive liquid-liquid microextraction coupled with capillary electrophoresis [7]. Multiclass determinations of additives in a single run were developed in recent year. Boyce [8] used micellar electrokinetic chromatography to separate and detect 6 antioxidants and 3 sweeteners in beverages. Ultra performance liquid chromatography (UPLC) coupled with electrospray ionization tandem mass spectrometry (ESI-MS/MS) was applied for rapid determination of 5 preservatives, 6 artificial sweeteners and 9 synthetic dyes in kimchi. The rapid separation was performed in $5.5 \mathrm{~min}$ and can be used for rapid quality control [9]. Methods focused on the sample pretreatment and cleanup were applied in accurate determination of preservatives and artificial sweeteners in juice by HPLC to remove matrix interference prior to chromatographic determination [10].

High resolution mass spectrometry (HRMS) such as orbitrap provided full-scan spectra with resolution 70,000 FWHM (full width at half maximum), which gave chance to look into the composition of a sample. The higher resolution made the assigned masses more accurate and background peaks better resolved. Studies had proved HRMS produced similar results in terms of reliability, specificity and sensitivity to the most wildly used low resolution MS/MS [11,12]. A validated approach analyzed 43 additives including sweeteners, flavor agents, antioxidants and preservatives in dairy product by quadrupole orbitrap mass spectrometry. Sample was prepared through quick, easy, cheap, effective, rugged and safe (QuEChERS) method prior to a 15min fast analysis [13]. Another similar approach described the detection of 69 dyes in wines [14]. The results proved HRMS was a powerful method for screening analytes in foods. Additional capabilities of HRMS beyond the current MS/MS were by confirming the presence of an analyte not included in the target list. The measure of analytes without previous compound-specific tuning and selection enabled retrospective data analysis for a broad list of compounds, so called nontargeted data acquisition [15]. Compared to tandem mass only applicable to around 200 targets [16], nontargeted data acquisition carried out larger compound database, and widely used as metabolic fingerprinting and profiling approaches [17]. Wang [18] generated a non-target data acquisition for target analysis called nDATA workflow for screening of 845 pesticide residues in fruits and vegetables by UHPLC/QOrbitrap. The detection was based on the retention time, mass accuracy of a precursor and fragment ions which significantly expanding the number of pesticides currently being screened by traditional MS/MS approaches.

The aim of this study was designed to build a screening method for 120 additives by HRMS. The major areas of routine monitoring of food were pesticide residues, veterinary residues and natural toxins. Methods for these three classes of residues were well developed for detection of multiple residues in a single commodity, and be able to quantitate. However, the multi-class analytical method for additives such as dyes, sweeteners, preservatives, antioxidants and flavoring agents was few. A nontargeted method called variable data independent acquisition (vDIA) was developed for comprehensive sample detection of 120 additives in this study.

\section{Methods}

\subsection{Chemicals}

Methanol, acetonitrile, ethyl acetate, acetone, formic acid and ammonia acetate were purchased from Sigma-Aldrich (St. Louis, MO, US). Reference standards including (name and purity\%) allura red AC $(98 \%)$, alizarin $(95 \%)$, alizarin green $(95 \%)$, amaranth $(95 \%)$, auramine $\mathrm{O}(95 \%)$, azorubine $(95 \%)$, benzyl violet $4 \mathrm{~B}(95 \%)$, brilliant blue FCF $(95 \%)$, carminic acid $(95 \%)$, chrysoidine G $(95 \%)$, citrus Red $2(95 \%)$, crocein orange G $(95 \%)$, curcumin $(95 \%)$, diethyl yellow $(95 \%)$, erythrosine $(95 \%)$, fast green FCF $(95 \%)$, indigo carmine $(95 \%)$, light green SF yellowish (95\%), lissamine green B (95\%), malachite green (95\%), metanil yellow (95\%), methyl yellow $(95 \%), \alpha$-naphthol orange $(95 \%)$, naphthol yellow S $(95 \%)$, new coccin $(95 \%)$, orange G $(80 \%)$, orange II $(95 \%)$, para red $(95 \%)$, patent blue V $(95 \%)$, phloxine $(95 \%)$, ponceau SX $(98 \%)$, quinoline yellow 
WS $(95 \%)$, rhodamine B $(95 \%)$, rose Bengal $(95 \%)$, scarlet GN (95\%), solvent green $3(95 \%)$, Sudan black B $(95 \%)$, Sudan I $(95 \%)$, Sudan II $(95 \%)$, Sudan III (95\%), Sudan IV (95\%), Sudan orange G $(95 \%)$, Sudan red 7B (95\%), Sudan red G $(95 \%)$, Sudan red B $(95 \%)$, sulforhodamine B $(95 \%)$, sunsent yellow FCF $(95 \%)$, tartrazine $(95 \%)$, xylene fast yellow $2 \mathrm{G}$ $(60 \%)$, pigment orange $5(98 \%)$, disperse orange 37 $(96 \%)$, oil orange SS $(98 \%)$, red $2 \mathrm{G}(98 \%)$, fumaric acid $(99 \%)$, succinic acid $(99 \%)$, L-glutamic acid $(99 \%)$, tartaric acid $(99 \%)$, sodium lactate $(99 \%)$, DLmalic acid $(99 \%)$, caffeine $(98 \%)$, L-theanine $(98 \%)$, glucono- $\delta$-lactone $(98 \%)$, propyl gallate $(98 \%)$, nordihydroguaiaretic acid $(97 \%)$, 4-hexylresorcinol $(98 \%)$, ethoxyquin $(99 \%)$, methyl p-hydroxybenzoate $(99 \%)$, ethyl p-hydroxybenzoate $(99 \%)$, propyl p-hydroxybenzoate $(99 \%)$, butyl p-hydroxybenzoate $(99 \%)$, benzyl 4-hydroxybenzoate $(99 \%)$, phenyl 4-hydroxybenzoate $(98 \%)$, n-heptyl 4hydroxybenzoate $(99 \%)$, natamycin $(99 \%)$, sorbic acid $(99 \%)$, aspartame $(98 \%)$, neohesperidin dihydrochalcone $(98 \%)$, neotame $(98 \%)$, rebaudioside A $(96 \%)$, rebaudioside B $(95 \%)$, sucralose $(98 \%)$, Lcysteine hydrochloride $(98 \%)$ and cyclamate $(100 \%$, SUPELCO) were from Sigma-Aldrich. Saccharin $(99 \%)$ and acesulfame potassium $(99 \%)$ were from Fluka (Charlotte, NC, US). Gentian violet $(100 \%)$, stevioside $(100 \%)$ and glycyrrhizic acid $(100 \%)$ were purchased from USP (Rockville, MD, US). Dulcin (99\%) and acid green 3 (unknown purity) were form Kanto-Kasel LTD. (Tokyo, Japan). Alitame (98\%) was from Toronto Research Chemicals (North York, ON, Canada). Eosin Y (85\%), fluorescein (95\%), brilliant yellow $(70 \%)$, disperse orange $3(96 \%)$, basic fuchsin (unknown purity), astrazon orange G (unknown purity), 2-methoxy-4-nitroaniline (98\%), alizarin yellow GG $(50 \%)$, fast yellow AB $(97 \%)$, disperse yellow $3(96 \%)$, o-aminoazotoluene $(99.3 \%)$, 1-(methylamino)-anthraquinone $(98 \%)$, 4-aminoazobenzene $(98.9 \%)$, lithol rubine BK (unknown purity) and new fuchsin (unknown purity) were from Uni-Onward Co. (Taipei, Taiwan). Astrazon orange R (unknown purity) was form TCI (Tokyo, Japan). Dyes with unknown purity of 4-amino carminic acid, solvent yellow 21, solvent orange 62, solvent red 8 and solvent yellow 16 were from BOC Sciences (Shirley, NY, US). Copper chlorophylls with unknown purity of $\mathrm{Cu}$ (II) chlorin $\mathrm{e} 4$ and $\mathrm{Cu}$ (II) chlorin e6 were from Frontier Scientific (Logan, UT, USA). Chemical each $100 \mathrm{mg}$ was dissolved in appropriate solvent (methanol, ethanol, acetonitrile, ethyl acetate or acetone) in a $10-\mathrm{mL}$ volumetric flask and diluted to volume as individual standard stock solution. Mixed solutions were prepared at 1, 5 or
$10 \mu \mathrm{g} / \mathrm{mL}$ in $50 \%$ methanol solution to generate target list of additives.

\subsection{Instrumentation for generating target list of additives}

An UHPLC-ESI-Orbitrap MS system consisted of an UltiMate 3,000 pump, a $Q$ Exactive mass spectrometer and a accucore $\mathrm{aQ}$ C18 column $(2.1 \times 150 \mathrm{~mm}, 2.6 \mu \mathrm{m}$ particle size, Thermo Fisher Scientific, Rockford, IL, US) were utilized. The column was maintained at $35^{\circ} \mathrm{C}$ and the flow rate was set at $0.5 \mathrm{~mL} / \mathrm{min}$. A gradient elution containing $0.1 \%$ formic acid with $20 \mathrm{mM}$ ammonium acetate (A), and acetonitrile with $0.1 \%$ formic acid (B) was applied. The gradient was hold on $1 \%$ (B) for first $1 \mathrm{~min}$ and then increased from 1 to $99 \%$ (B) over the next $8 \mathrm{~min}$. The eluent was remained for the next $7 \mathrm{~min}$, and then mobile phase (B) was retained to $1 \%$ over the next $0.1 \mathrm{~min}$, and this was followed by a $2.9 \mathrm{~min}$ re-equilibration period at $1 \%$ (B) prior to the next injection. The injection volume was $5 \mu \mathrm{L}$. The mass spectrometer was operated at ESI positive $(3.5 \mathrm{kV})$ and negative $(2.5 \mathrm{kV})$ mode. Resolution was set at 70,000 (defined at $m / z=200$ and was set at full width at half maximum, FWHM). The precursor list was built by direct infusion mass spectrometry of each individual additive standard at concentration of $1 \mathrm{ppm}$.

\subsection{Evaluation of limits of detection (LODs) by data independent acquisition (DIA)}

Candy and carbonated sparkling water were used as blank sample for the evaluation of LODs for 79 colorants. Pork jerky and carbonated sparkling water were used for 13 sweeteners. Twelve preservatives were tested in pork jerky. Seven antioxidants and 9 flavoring agents were evaluated in carbonated sparkling water. Carbonated sparkling water was ultrasonicated for $15 \mathrm{~min}$, and $10 \mathrm{~g}$ was transferred into a $100-\mathrm{mL}$ volumetric flask. Additive standard stock solutions were spiked separately at the concentration $0.01,0.02,0.05,0.1,0.2,0.5,1,5$ and $10 \mathrm{mg} / \mathrm{kg}$, and made up to volume with $50 \%$ methanol solution $(\mathrm{v} / \mathrm{v})$. Solutions were filtered with membrane filters prior to analysis. Candy and pork jerky were ground separately and $10 \mathrm{~g}$ each was transferred into a $100-\mathrm{mL}$ volumetric flask. Additive standard stock solutions were then spiked into sample for $30 \mathrm{~min}$, and made up to volume with $50 \%$ methanol solution. The volumetric flask was untrasoncated for $15 \mathrm{~min}$, and then centrifuged at $5,000 \times g$. The filtrate was passed through a polyvinylidene fluoride (PVDF) membrane filter as sample solution. 
Mass spectrometer worked in DIA mode and the parameters were as following. A full scan form $\mathrm{m} / \mathrm{z}$ $70-1,050$ was conducted, and then precursors in wide windows were selected to obtain $21 \mathrm{MS} / \mathrm{MS}$ spectra. Isolation window 27 amu was applied for $m /$ $z 100$ to 500 in every 25 amu increment (eg. 100-125, 125-150, ..., 475-500 amu). Isolation window 104 amu was utilized for $\mathrm{m} / \mathrm{z} 500$ to 1,000 in every 100 amu increment. The ions in every collection were then sent to high energy dissociation (HCD) cell for fragmentation, and the followed C-trap prior to orbitrap analysis. The cycle time for a full scan and 21 fragments spectra was about $2.6 \mathrm{~s}$. The samples were required to run HPLC HRMS/MS twice, one for positive ESI and another for negative ESI due to the long cycle time of data acquisition.

\subsection{Surveillance}

Candies, beverages and soy sauces in total 33 samples were purchase from local market. Samples were separated into liquid and solid samples, and followed the same procedures described in sample preparation for LODs evaluation. The detection and data processing of non-target data acquisition for target analysis were performed using TraceFinder 3.3 software (Thermo Fisher Scitific). In a positive sample, the precursor ion of an analyte was extracted form full scan spectra, and the product ions were extracted form fragment spectra associated to the precursor segment.

\section{Results and discussion}

The precursor ion and two product ions along with retention time of each analyte were shown in Table 1. The ionization of general chemicals were either $[\mathrm{M}+\mathrm{H}]^{+}$or $[\mathrm{M}-\mathrm{H}]^{-}$. However, for colorants, some were exceptional. [M $+\mathrm{H}-\mathrm{Na}]^{-},[\mathrm{M}-\mathrm{Na}]^{-}$, $\left[\mathrm{M}+\mathrm{H}_{2}-\mathrm{Na}\right]^{+}, \quad\left[\mathrm{M}+\mathrm{H}_{3}-\mathrm{Na}_{2}\right]^{-}, \quad\left[\mathrm{M}+\mathrm{H}_{3}-\mathrm{Na}\right]^{-}$, $[\mathrm{M}-\mathrm{Cl}]^{+}$and $[\mathrm{M}+\mathrm{H}-\mathrm{Ca}]^{-}$might happen depended on the chemicals and the $\mathrm{pH}$ of sample solution and eluent. The target list was generated in parallel reaction monitoring (PRM) mode of mass spectrometry. A full scan at scan range $m / z 70-1,050$ was first conducted, and then the scan data was compared with the established precursor list. If there were a match, the mass spectrometer would isolate a target precursor ion (window was $4 \mathrm{amu}$ ), then fragmented the targeted precursor ion in the collision cell $(30 \mathrm{eV}$ and $60 \mathrm{eV}$ stored in C-trap), and then sent to Orbitrap for detection. The resulting product ions were obtained as MS/MS spectrum, and all spectra of standard compounds were listed in the supplemental file (https://www.jfda-online.com/cgi/editor. cgi article $=3366 \&$ window $=$ additional

files\&context=journal). The dead time, no interaction between the sample and the column, was estimated to be $0.7 \mathrm{~min}\left[(0.105 \mathrm{~cm})^{2} \times \pi \times 15 \mathrm{~cm} \times 70 \%\right.$ / $0.5 \mathrm{~cm}^{3}$ per $\min , 70 \%$ was an estimate of interstitial void space in porous silica particles]. Most additives obtained good retentions, except flavoring agents such as L-cysteine, fumaric acid, L-glutamic acid, tartaric acid, sodium lactate, DL-malic acid, glucono- $\delta$-lactone, which obtained retention time between 0.6 and $0.8 \mathrm{~min}$. These compounds co-eluted with other no retention food matrix. The mass spectrometer worked as the secondary separation tool. The low-resolution mass spectrometer (LRMS) basically separate two ions differing by one mass unit along the whole range scanned. The high-resolution mass spectrometer (HRMS), such as Orbitrap, measures ion oscillation frequencies and transfer into mass-to-charge ratio $(\mathrm{m} / \mathrm{z})$ with resolution 70,000 FWHM. The instrument provides accurate mass with detecting error below $5 \mathrm{ppm}$ which enable the secondary separation of analytes in complex co-elute food matrix. Fig. $1 b$ showed a chromatogram (total ion chromatogram, TIC) of a soy sauce (sample 32 ) at RT between 0 and $2.5 \mathrm{~min}$. There was a big and broad peak indicated no/poor retention and co-elute in the first $2 \mathrm{~min}$. The ESI negative spectrum of $\mathrm{RT}=0.7$ was showed in Fig. $1 \mathrm{a}$ that dressed there were a few compounds co-elute around the column dead time. These compounds were high polar chemicals performed no retention on a typical C18 column. However, lactate (Fig. 1c) and glutamic acid (Fig. 1d) were able to be separated from the co-elute matrix by selecting theoretical accurate masses of 89.0244 and 146.0459, respectively. HRMS was powerful and enable the secondary separation of analytes from very complex coelute without interference. In Fig. 1a, obtained accurate mass of lactate $\mathrm{m} / \mathrm{z} 89.02403$ and glutamic acid $\mathrm{m} / \mathrm{z} 146.04531$ showed differences of -4.15 and $-4.04 \mathrm{ppm}$, respectively, to their assigned value in Table 1. The advantage of HRMS enabled the detection and separation of analytes even in dead time range of chromatographic separation.

\subsection{DIA for precursor selection and product ions detection}

DIA required no selection of a special precursor in a narrow range of typically 1 amu (conventional MRM), instead a wide window such as 27 or 104 amu in this study was selected. Various wide windows were selected to cover all potential analytes in the range between $\mathrm{m} / \mathrm{z} 100$ to $\mathrm{m} / \mathrm{z} 1,000$, and the followed fragmentation generated product ions for 
Table 1. The ionization mode, detection ions, retention time and LODs of target compounds were determined.

\begin{tabular}{|c|c|c|c|c|c|c|c|c|c|}
\hline & \multirow[t]{2}{*}{ Compound } & \multirow{2}{*}{$\begin{array}{l}\text { Elemental } \\
\text { composition }\end{array}$} & \multirow{2}{*}{$\begin{array}{l}\text { Ionization } \\
\text { mode }\end{array}$} & \multirow{2}{*}{$\begin{array}{l}\text { Precursor Ion } \\
\text { (theoretical) }\end{array}$} & \multirow{2}{*}{$\begin{array}{l}\text { Delta } \\
\text { mass }^{a}\end{array}$} & \multirow{2}{*}{$\begin{array}{l}\text { Product } \\
\text { ions }\end{array}$} & \multirow{2}{*}{$\begin{array}{l}\text { Retention } \\
\text { time }\end{array}$} & \multicolumn{2}{|c|}{ LODs (ppm) } \\
\hline & & & & & & & & Candy & Beverage \\
\hline 1 & Tartrazine & $\mathrm{C}_{16} \mathrm{H}_{9} \mathrm{~N}_{4} \mathrm{Na}_{3} \mathrm{O}_{9} \mathrm{~S}_{2}$ & {$[\mathrm{M}+2 \mathrm{H}-3 \mathrm{Na}]-$} & 466.9973 & 2.7 & $\begin{array}{l}197.9872 \\
172.0068\end{array}$ & 3.02 & 0.5 & 0.5 \\
\hline 2 & Amaranth & $\mathrm{C}_{20} \mathrm{H}_{11} \mathrm{~N}_{2} \mathrm{Na}_{3} \mathrm{O}_{10} \mathrm{~S}_{3}$ & {$[\mathrm{M}+2 \mathrm{H}-3 \mathrm{Na}]-$} & 536.9738 & 2.4 & $\begin{array}{l}316.9669 \\
237.0101\end{array}$ & 3.17 & 0.5 & 0.5 \\
\hline 3 & Indigo carmine & $\mathrm{C}_{16} \mathrm{H}_{8} \mathrm{~N}_{2} \mathrm{Na}_{2} \mathrm{O}_{8} \mathrm{~S}_{2}$ & {$[\mathrm{M}+\mathrm{H}-2 \mathrm{Na}] 2-$} & 209.9867 & 6.7 & $\begin{array}{l}79.9559 \\
154.9584\end{array}$ & 3.38 & 5 & 5 \\
\hline 4 & $\begin{array}{l}\text { Cochineal red A, } \\
\text { Ponceau } 4 \mathrm{R}\end{array}$ & $\mathrm{C}_{20} \mathrm{H}_{11} \mathrm{~N}_{2} \mathrm{Na}_{3} \mathrm{O}_{10} \mathrm{~S}_{3}$ & {$[\mathrm{M}+2 \mathrm{H}-3 \mathrm{Na}] 2-$} & 267.9833 & 3.2 & $\begin{array}{l}145.0280 \\
208.9909\end{array}$ & 3.48 & 5 & 5 \\
\hline 5 & Sunset yellow & $\mathrm{C}_{16} \mathrm{H}_{10} \mathrm{~N}_{2} \mathrm{Na}_{2} \mathrm{O}_{7} \mathrm{~S}_{2}$ & {$[\mathrm{M}+\mathrm{H}-2 \mathrm{Na}]-$} & 407.0013 & 1.4 & $\begin{array}{l}205.9917 \\
327.0445\end{array}$ & 3.68 & 0.5 & 0.5 \\
\hline 6 & Naphthol yellow S & $\mathrm{C}_{10} \mathrm{H}_{4} \mathrm{~N}_{2} \mathrm{Na}_{2} \mathrm{O}_{8} \mathrm{~S}$ & {$[\mathrm{M}+\mathrm{H}-2 \mathrm{Na}]-$} & 312.9772 & 1.3 & $\begin{array}{l}233.0203 \\
295.9744\end{array}$ & 3.68 & 0.5 & 0.5 \\
\hline 7 & Orange G & $\mathrm{C}_{16} \mathrm{H}_{10} \mathrm{~N}_{2} \mathrm{Na}_{2} \mathrm{O}_{7} \mathrm{~S}_{2}$ & {$[\mathrm{M}+\mathrm{H}-2 \mathrm{Na}]-$} & 407.0013 & 1.7 & $\begin{array}{l}301.9560 \\
158.0373\end{array}$ & 3.74 & 0.1 & 0.1 \\
\hline 8 & Allura red AC & $\mathrm{C}_{18} \mathrm{H}_{14} \mathrm{~N}_{2} \mathrm{Na}_{2} \mathrm{O}_{8} \mathrm{~S}_{2}$ & {$[\mathrm{M}+\mathrm{H}-2 \mathrm{Na}]-$} & 451.0275 & 1.6 & $\begin{array}{l}205.9917 \\
79.9559\end{array}$ & 3.95 & 0.5 & 0.5 \\
\hline 9 & Carminic Acid & $\mathrm{C}_{22} \mathrm{H}_{20} \mathrm{O}_{13}$ & {$[\mathrm{M}-\mathrm{H}]-$} & 491.0831 & 1.2 & $\begin{array}{l}357.0610 \\
327.0510\end{array}$ & 4.06 & 0.5 & 0.5 \\
\hline 10 & $\begin{array}{l}\text { Xylene fast } \\
\text { yellow } 2 G\end{array}$ & $\mathrm{C}_{16} \mathrm{H}_{10} \mathrm{Cl}_{2} \mathrm{~N}_{4} \mathrm{Na}_{2} \mathrm{O}_{7} \mathrm{~S}_{2}$ & {$[\mathrm{M}+\mathrm{H}-2 \mathrm{Na}] 2-$} & 251.9689 & 1.7 & $\begin{array}{l}170.9996 \\
107.0377\end{array}$ & 4.1 & 5 & 5 \\
\hline 11 & Scarlet GN & $\mathrm{C}_{18} \mathrm{H}_{14} \mathrm{~N}_{2} \mathrm{Na}_{2} \mathrm{O}_{7} \mathrm{~S}_{2}$ & {$[\mathrm{M}+\mathrm{H}-2 \mathrm{Na}]+$} & 437.0472 & 2.3 & $\begin{array}{l}201.0454 \\
118.0651\end{array}$ & 4.23 & 0.1 & 0.1 \\
\hline 12 & Azorubine & $\mathrm{C}_{20} \mathrm{H}_{12} \mathrm{~N}_{2} \mathrm{Na}_{2} \mathrm{O}_{7} \mathrm{~S}_{2}$ & {$[\mathrm{M}+\mathrm{H}-2 \mathrm{Na}]-$} & 457.0170 & 3.9 & $\begin{array}{l}377.0601 \\
221.0151\end{array}$ & 4.41 & 0.5 & 0.5 \\
\hline 13 & Ponceau SX & $\mathrm{C}_{18} \mathrm{H}_{14} \mathrm{~N}_{2} \mathrm{Na}_{2} \mathrm{O}_{7} \mathrm{~S}_{2}$ & {$[\mathrm{M}+\mathrm{H}-2 \mathrm{Na}]^{-}$} & 435.0326 & 2.8 & $\begin{array}{l}355.0748 \\
199.0308\end{array}$ & 4.46 & 0.1 & 0.1 \\
\hline 14 & Light green SF & $\mathrm{C}_{37} \mathrm{H}_{34} \mathrm{~N}_{2} \mathrm{Na}_{2} \mathrm{O}_{9} \mathrm{~S}_{3}$ & {$[\mathrm{M}+\mathrm{H}-2 \mathrm{Na}]^{-}$} & 747.1510 & 1.1 & $\begin{array}{l}683.1891 \\
170.0043\end{array}$ & 4.49 & 0.5 & 0.5 \\
\hline 15 & G3 (Fast green FCF & $\mathrm{C}_{37} \mathrm{H}_{34} \mathrm{~N}_{2} \mathrm{Na}_{2} \mathrm{O}_{10} \mathrm{~S}_{3}$ & {$[\mathrm{M}+\mathrm{H}-2 \mathrm{Na}]^{-}$} & 763.1459 & 1.4 & $\begin{array}{l}497.1547 \\
577.1115\end{array}$ & 4.53 & 0.5 & 0.5 \\
\hline 16 & Lissamine green B & $\mathrm{C}_{27} \mathrm{H}_{25} \mathrm{~N}_{2} \mathrm{NaO}_{7} \mathrm{~S}_{2}$ & {$[\mathrm{M}+2 \mathrm{H}-\mathrm{Na}]^{+}$} & 555.1254 & 1.5 & $\begin{array}{l}392.1883 \\
377.1648\end{array}$ & 4.56 & 0.5 & 0.5 \\
\hline 17 & Brilliant blue FCF & $\mathrm{C}_{37} \mathrm{H}_{34} \mathrm{~N}_{2} \mathrm{Na}_{2} \mathrm{O}_{9} \mathrm{~S}_{3}$ & {$[\mathrm{M}+2 \mathrm{H}-2 \mathrm{Na}]^{-}$} & 747.1510 & 2.6 & $\begin{array}{l}561.1166 \\
260.0512\end{array}$ & 4.6 & 0.5 & 0.5 \\
\hline 18 & $\alpha$-Naphthol orange & $\mathrm{C}_{16} \mathrm{H}_{11} \mathrm{~N}_{2} \mathrm{NaO}_{4} \mathrm{~S}$ & {$[\mathrm{M}-\mathrm{Na}]^{-}$} & 327.0445 & 2.4 & $\begin{array}{l}170.9995 \\
247.0876\end{array}$ & 4.76 & 0.1 & 0.1 \\
\hline 19 & Quinoline yellow S & $\mathrm{C}_{18} \mathrm{H}_{13} \mathrm{NaNO}_{5} \mathrm{~S}$ & {$[\mathrm{M}-3 \mathrm{H}-\mathrm{Na}]^{-}$} & 352.0285 & 2.7 & $\begin{array}{l}288.0666 \\
272.0717\end{array}$ & 4.77 & 0.1 & 0.1 \\
\hline 20 & Sulforhodamine B & $\mathrm{C}_{27} \mathrm{H}_{30} \mathrm{~N}_{2} \mathrm{O}_{7} \mathrm{~S}_{2}$ & {$[\mathrm{M}+\mathrm{H}]^{+}$} & 559.1567 & 2.5 & $\begin{array}{l}515.0941 \\
501.0910\end{array}$ & 5.21 & 0.05 & 0.05 \\
\hline 21 & Crocein orange $G$ & $\mathrm{C}_{16} \mathrm{H}_{11} \mathrm{~N}_{2} \mathrm{NaO}_{4} \mathrm{~S}$ & {$[\mathrm{M}-\mathrm{Na}]^{-}$} & 327.0445 & 2.6 & $\begin{array}{l}206.9995 \\
142.0298\end{array}$ & 5.26 & 0.1 & 0.1 \\
\hline 22 & $\begin{array}{l}\text { Orange II } \\
\text { (Acid orange 7) }\end{array}$ & $\mathrm{C}_{16} \mathrm{H}_{11} \mathrm{~N}_{2} \mathrm{NaSO}_{4}$ & {$[\mathrm{M}-\mathrm{Na}]^{-}$} & 327.0445 & 1.5 & $\begin{array}{l}170.9995 \\
155.9874\end{array}$ & 5.26 & 0.1 & 0.1 \\
\hline 23 & Patent blue V & $\mathrm{C}_{27} \mathrm{H}_{31} \mathrm{~N}_{2} \mathrm{NaO}_{7} \mathrm{~S}_{2}$ & {$[\mathrm{M}+2 \mathrm{H}-\mathrm{Na}]^{+}$} & 561.1724 & 2.8 & $\begin{array}{l}479.1999 \\
435.1346\end{array}$ & 5.28 & 0.5 & 0.5 \\
\hline 24 & Alizarin & $\mathrm{C}_{14} \mathrm{H}_{8} \mathrm{O}_{4}$ & {$[\mathrm{M}-\mathrm{H}]^{-}$} & 239.0350 & 3.8 & $\begin{array}{l}211.0400 \\
195.0451\end{array}$ & 5.42 & 0.5 & 0.5 \\
\hline 25 & $\begin{array}{l}\text { Alizarin green } \\
\text { (Patent green) }\end{array}$ & $\mathrm{C}_{37} \mathrm{H}_{34} \mathrm{ClN}_{2} \mathrm{NaO}_{6} \mathrm{~S}_{2}$ & {$[\mathrm{M}+2 \mathrm{H}-\mathrm{Na}]^{+}$} & 703.1698 & 2.5 & $\begin{array}{l}517.1342 \\
533.1655\end{array}$ & 5.48 & 0.5 & 0.5 \\
\hline 26 & Chrysoidine G & $\mathrm{C}_{12} \mathrm{H}_{12} \mathrm{~N}_{4} \cdot \mathrm{HCl}$ & {$[\mathrm{M}-\mathrm{Cl}]^{+}$} & 213.1135 & 4.2 & $\begin{array}{l}121.0634 \\
94.0525\end{array}$ & 5.49 & 0.1 & 0.1 \\
\hline 27 & Curcumin & $\mathrm{C}_{21} \mathrm{H}_{20} \mathrm{O}_{6}$ & {$[\mathrm{M}-\mathrm{H}]^{-}$} & 367.1187 & 6.7 & $\begin{array}{l}134.0368 \\
173.0608\end{array}$ & 5.8 & 5 & 5 \\
\hline 28 & Benzyl violet $4 \mathrm{~B}$ & $\mathrm{C}_{39} \mathrm{H}_{40} \mathrm{~N}_{3} \mathrm{NaO}_{6} \mathrm{~S}_{2}$ & {$[\mathrm{M}-\mathrm{Na}]^{-}$} & 710.2364 & 2.7 & $\begin{array}{l}630.2810 \\
540.2310\end{array}$ & 5.8 & 0.1 & 0.1 \\
\hline 29 & Auramine $\mathrm{O}$ & $\mathrm{C}_{17} \mathrm{H}_{21} \mathrm{~N}_{3}$ & {$[\mathrm{M}+\mathrm{H}]^{+}$} & 268.1808 & 1.2 & $\begin{array}{l}147.0916 \\
131.0603\end{array}$ & 5.99 & 0.1 & 0.1 \\
\hline 30 & R7 (Erythrosine) & $\mathrm{C}_{20} \mathrm{H}_{6} \mathrm{I}_{4} \mathrm{Na}_{2} \mathrm{O}_{5}$ & {$[\mathrm{M}+3 \mathrm{H}-2 \mathrm{Na}]^{-}$} & 836.6623 & 3.1 & $\begin{array}{l}582.8521 \\
329.0435\end{array}$ & 6.4 & 0.5 & 0.5 \\
\hline
\end{tabular}


Table 1. (continued)

\begin{tabular}{|c|c|c|c|c|c|c|c|c|c|}
\hline & \multirow[t]{2}{*}{ Compound } & \multirow{2}{*}{$\begin{array}{l}\text { Elemental } \\
\text { composition }\end{array}$} & \multirow{2}{*}{$\begin{array}{l}\text { Ionization } \\
\text { mode }\end{array}$} & \multirow{2}{*}{$\begin{array}{l}\text { Precursor Ion } \\
\text { (theoretical) }\end{array}$} & \multirow{2}{*}{$\begin{array}{l}\text { Delta } \\
\text { mass }^{a}\end{array}$} & \multirow{2}{*}{$\begin{array}{l}\text { Product } \\
\text { ions }\end{array}$} & \multirow{2}{*}{$\begin{array}{l}\text { Retention } \\
\text { time }\end{array}$} & \multicolumn{2}{|c|}{ LODs (ppm) } \\
\hline & & & & & & & & Candy & Beverage \\
\hline 31 & Sudan orange $\mathrm{G}$ & $\mathrm{C}_{12} \mathrm{H}_{10} \mathrm{~N}_{2} \mathrm{O}_{2}$ & {$[\mathrm{M}+\mathrm{H}]^{+}$} & 215.0815 & 2.5 & $\begin{array}{l}93.0573 \\
95.0127\end{array}$ & 7.09 & 0.1 & 0.1 \\
\hline 32 & Malachite green & $\mathrm{C}_{23} \mathrm{H}_{25} \mathrm{ClN}_{2}$ & {$[\mathrm{M}-\mathrm{Cl}]^{+}$} & 329.2012 & 4.9 & $\begin{array}{l}313.1699 \\
208.1120\end{array}$ & 7.52 & 0.5 & 0.5 \\
\hline 33 & Rhodamine B & $\mathrm{C}_{28} \mathrm{H}_{31} \mathrm{ClN}_{2} \mathrm{O}_{3}$ & {$[\mathrm{M}-\mathrm{Cl}]^{+}$} & 443.2329 & 1.4 & $\begin{array}{l}399.1703 \\
355.1077\end{array}$ & 7.58 & 0.05 & 0.05 \\
\hline 34 & Phloxine & $\mathrm{C}_{20} \mathrm{H}_{2} \mathrm{Br}_{4} \mathrm{Cl}_{4} \mathrm{Na}_{2} \mathrm{O}_{5}$ & {$[\mathrm{M}-\mathrm{Na}]^{-}$} & 784.5406 & 4.4 & $\begin{array}{l}658.6242 \\
704.5731\end{array}$ & 7.72 & 5 & 5 \\
\hline 35 & Rose bengal & $\mathrm{C}_{20} \mathrm{H}_{2} \mathrm{Cl}_{4} \mathrm{I}_{4} \mathrm{Na}_{2} \mathrm{O}_{5}$ & {$[\mathrm{M}-\mathrm{Na}]^{-}$} & 970.4919 & 2.9 & $\begin{array}{l}672.6936 \\
890.5253\end{array}$ & 7.75 & 5 & 5 \\
\hline 36 & Para red & $\mathrm{C}_{16} \mathrm{H}_{11} \mathrm{~N}_{3} \mathrm{O}_{3}$ & {$[\mathrm{M}+\mathrm{H}]^{+}$} & 294.0873 & 1.4 & $\begin{array}{l}277.0843 \\
128.0494\end{array}$ & 8.53 & 0.5 & $-{ }^{b}$ \\
\hline 37 & Methyl yellow & $\mathrm{C}_{14} \mathrm{H}_{15} \mathrm{~N}_{3}$ & {$[\mathrm{M}+\mathrm{H}]^{+}$} & 226.1339 & 2.7 & $\begin{array}{l}95.0494 \\
105.0447\end{array}$ & 8.54 & 0.1 & - \\
\hline 38 & Sudan red G & $\mathrm{C}_{17} \mathrm{H}_{14} \mathrm{~N}_{2} \mathrm{O}_{2}$ & {$[\mathrm{M}+\mathrm{H}]^{+}$} & 279.1128 & 4.0 & $\begin{array}{l}123.0678 \\
108.0443\end{array}$ & 8.88 & 0.1 & 0.1 \\
\hline 39 & Citrus Red 2 & $\mathrm{C}_{18} \mathrm{H}_{16} \mathrm{~N}_{2} \mathrm{O}_{3}$ & {$[\mathrm{M}+\mathrm{H}]^{+}$} & 309.1234 & 2.6 & $\begin{array}{l}138.0549 \\
153.0784\end{array}$ & 8.9 & 0.5 & 0.5 \\
\hline 40 & Sudan I & $\mathrm{C}_{16} \mathrm{H}_{12} \mathrm{~N}_{2} \mathrm{O}$ & {$[\mathrm{M}+\mathrm{H}]^{+}$} & 249.1022 & 1.2 & $\begin{array}{l}128.0494 \\
232.0992\end{array}$ & 8.94 & 0.5 & - \\
\hline 41 & Dimethyl yellow & $\mathrm{C}_{16} \mathrm{H}_{19} \mathrm{~N}_{3}$ & {$[\mathrm{M}+\mathrm{H}]^{+}$} & 254.1652 & 2.7 & $\begin{array}{l}95.0494 \\
134.0943\end{array}$ & 9.05 & 0.1 & - \\
\hline 42 & Sudan II & $\mathrm{C}_{18} \mathrm{H}_{16} \mathrm{~N}_{2} \mathrm{O}$ & {$[\mathrm{M}+\mathrm{H}]^{+}$} & 277.1335 & 3.2 & $\begin{array}{l}121.0886 \\
106.0651\end{array}$ & 9.95 & 5 & 5 \\
\hline 43 & Sudan black B & $\mathrm{C}_{29} \mathrm{H}_{24} \mathrm{~N}_{6}$ & {$[\mathrm{M}+\mathrm{H}]^{+}$} & 457.2135 & 4.5 & $\begin{array}{l}193.0760 \\
211.1104\end{array}$ & 10.28 & 5 & 5 \\
\hline 44 & Sudan III & $\mathrm{C}_{22} \mathrm{H}_{16} \mathrm{~N}_{4} \mathrm{O}$ & {$[\mathrm{M}+\mathrm{H}]{ }^{+}$} & 353.1397 & 2.9 & $\begin{array}{l}196.0869 \\
128.0494\end{array}$ & 10.69 & 0.5 & - \\
\hline 45 & Solvent green 3 & $\mathrm{C}_{28} \mathrm{H}_{22} \mathrm{~N}_{2} \mathrm{O}_{2}$ & {$[\mathrm{M}+\mathrm{H}]^{+}$} & 419.1754 & 2.3 & $\begin{array}{l}327.1128 \\
401.1648\end{array}$ & 10.79 & 0.5 & - \\
\hline 46 & Sudan red 7B & $\mathrm{C}_{24} \mathrm{H}_{21} \mathrm{~N}_{5}$ & {$[\mathrm{M}+\mathrm{H}]^{+}$} & 380.1870 & 2.7 & $\begin{array}{l}169.0760 \\
183.0916\end{array}$ & 10.95 & 5 & 5 \\
\hline 47 & Sudan IV & $\mathrm{C}_{24} \mathrm{H}_{20} \mathrm{~N}_{4} \mathrm{O}$ & {$[\mathrm{M}+\mathrm{H}]^{+}$} & 381.1710 & 3.8 & $\begin{array}{l}224.1175 \\
276.1121\end{array}$ & 11.46 & 0.5 & - \\
\hline 48 & Metanil yellow & $\mathrm{C}_{18} \mathrm{H}_{14} \mathrm{~N}_{3} \mathrm{NaO}_{3} \mathrm{~S}$ & {$[\mathrm{M}-\mathrm{Na}]^{-}$} & 352.0761 & 0.9 & $\begin{array}{l}171.9817 \\
155.9881\end{array}$ & 5.77 & 0.1 & 0.1 \\
\hline 49 & Eosin $Y$ & $\mathrm{C}_{20} \mathrm{H}_{8} \mathrm{Br}_{4} \mathrm{Na}_{2} \mathrm{O}_{5}$ & {$[\mathrm{M}+\mathrm{H}-2 \mathrm{Na}]^{-}$} & 646.6992 & 0.6 & $\begin{array}{l}522.7816 \\
442.8743\end{array}$ & 6.26 & 10 & 10 \\
\hline 50 & Fluorescein & $\mathrm{C}_{20} \mathrm{H}_{12} \mathrm{O}_{5}$ & {$[\mathrm{M}-\mathrm{H}]^{-}$} & 331.0612 & 0.9 & $\begin{array}{l}286.0635 \\
243.0815\end{array}$ & 5.8 & 0.2 & 0.2 \\
\hline 51 & Brilliant yellow & $\mathrm{C}_{26} \mathrm{H}_{18} \mathrm{~N}_{4} \mathrm{Na}_{2} \mathrm{O}_{8} \mathrm{~S}_{2}$ & {$[\mathrm{M}+\mathrm{H}-2 \mathrm{Na}]^{-}$} & 579.065 & 0.4 & $\begin{array}{l}499.1082 \\
369.9836\end{array}$ & 4.59 & 10 & 10 \\
\hline 52 & Disperse orange 3 & $\mathrm{C}_{12} \mathrm{H}_{10} \mathrm{~N}_{4} \mathrm{O}_{2}$ & {$[\mathrm{M}-\mathrm{H}]^{-}$} & 241.0731 & 1.5 & $\begin{array}{l}122.0248 \\
211.0751\end{array}$ & 7.22 & 0.5 & 0.5 \\
\hline 53 & $\begin{array}{l}\text { Gentian violet } \\
\text { (Crystal violet) }\end{array}$ & $\mathrm{C}_{25} \mathrm{H}_{30} \mathrm{~N}_{3} \mathrm{Cl}$ & {$[\mathrm{M}-\mathrm{Cl}]^{+}$} & 372.2434 & 1.1 & $\begin{array}{l}356.2121 \\
340.1808\end{array}$ & 7.89 & 0.1 & 0.1 \\
\hline 54 & Basic fuchsin & $\mathrm{C}_{20} \mathrm{H}_{20} \mathrm{ClN}_{3}$ & {$[\mathrm{M}-\mathrm{Cl}]^{+}$} & 302.1652 & 1.0 & $\begin{array}{l}209.1072 \\
195.0917\end{array}$ & 5.38 & 0.1 & 0.2 \\
\hline 55 & Astrazon orange $G$ & $\mathrm{C}_{22} \mathrm{H}_{23} \mathrm{ClN}_{2}$ & {$[\mathrm{M}-\mathrm{Cl}]^{+}$} & 315.1856 & 1.6 & $\begin{array}{l}300.1617 \\
285.1386\end{array}$ & 6.31 & 0.1 & 0.1 \\
\hline 56 & Astrazon orange $\mathrm{R}$ & $\mathrm{C}_{28} \mathrm{H}_{27} \mathrm{ClN}_{2}$ & {$[\mathrm{M}-\mathrm{Cl}]^{+}$} & 391.2169 & 1.9 & $\begin{array}{l}376.1934 \\
361.1699\end{array}$ & 7.53 & 0.1 & 0.1 \\
\hline 57 & Basic violet 2 & $\mathrm{C}_{22} \mathrm{H}_{24} \mathrm{~N}_{3} \mathrm{Cl}$ & {$[\mathrm{M}-\mathrm{Cl}]^{+}$} & 330.1965 & 1.8 & $\begin{array}{l}300.1495 \\
223.123\end{array}$ & 5.93 & 0.1 & 0.1 \\
\hline 58 & $\begin{array}{l}\text { 2-Methoxy-4-nitroaniline } \\
\text { (Fast red B Base) }\end{array}$ & $\mathrm{C}_{7} \mathrm{H}_{8} \mathrm{~N}_{2} \mathrm{O}_{3}$ & {$[\mathrm{M}+\mathrm{H}]^{+}$} & 169.0608 & 1.2 & $\begin{array}{l}152.058 \\
72.0444\end{array}$ & 5.26 & 0.2 & 0.2 \\
\hline 59 & Alizarin yellow GG & $\mathrm{C}_{13} \mathrm{H}_{8} \mathrm{~N}_{3} \mathrm{NaO}_{5}$ & {$[\mathrm{M}-\mathrm{Na}]^{-}$} & 286.0469 & 0.9 & $\begin{array}{l}242.0571 \\
156.0581\end{array}$ & 6.23 & 0.1 & 0.1 \\
\hline 60 & Fast yellow $\mathrm{AB}$ & $\mathrm{C}_{12} \mathrm{H}_{11} \mathrm{~N}_{3} \mathrm{O}_{6} \mathrm{~S}_{2}$ & {$[\mathrm{M}-\mathrm{H}]^{-}$} & 356.0016 & 0.6 & $\begin{array}{l}276.0448 \\
248.0387\end{array}$ & 3.1 & 10 & 10 \\
\hline
\end{tabular}


Table 1. (continued)

\begin{tabular}{|c|c|c|c|c|c|c|c|c|c|}
\hline & \multirow[t]{2}{*}{ Compound } & \multirow{2}{*}{$\begin{array}{l}\text { Elemental } \\
\text { composition }\end{array}$} & \multirow{2}{*}{$\begin{array}{l}\text { Ionization } \\
\text { mode }\end{array}$} & \multirow{2}{*}{$\begin{array}{l}\text { Precursor Ion } \\
\text { (theoretical) }\end{array}$} & \multirow{2}{*}{$\begin{array}{l}\text { Delta } \\
\text { mass }^{a}\end{array}$} & \multirow{2}{*}{$\begin{array}{l}\text { Product } \\
\text { ions }\end{array}$} & \multirow{2}{*}{$\begin{array}{l}\text { Retention } \\
\text { time }\end{array}$} & \multicolumn{2}{|c|}{ LODs (ppm) } \\
\hline & & & & & & & & Candy & Beverage \\
\hline 61 & Disperse yellow 3 & $\mathrm{C}_{15} \mathrm{H}_{15} \mathrm{~N}_{3} \mathrm{O}_{2}$ & {$[\mathrm{M}-\mathrm{H}]^{-}$} & 268.1092 & 0.7 & $\begin{array}{l}134.0611 \\
92.0506\end{array}$ & 7.31 & 0.1 & 0.1 \\
\hline 62 & o-Aminoazotoluene & $\mathrm{C}_{14} \mathrm{H}_{15} \mathrm{~N}_{3}$ & {$[\mathrm{M}+\mathrm{H}]^{+}$} & 226.1339 & 1.5 & $\begin{array}{l}121.076 \\
91.0542\end{array}$ & 7.74 & 0.1 & 0.1 \\
\hline 63 & Pigment orange 5 & $\mathrm{C}_{16} \mathrm{H}_{10} \mathrm{~N}_{4} \mathrm{O}_{5}$ & {$[\mathrm{M}-\mathrm{H}]^{-}$} & 337.0578 & 1.2 & $\begin{array}{l}125.0118 \\
167.0098\end{array}$ & 8.11 & 0.5 & 0.5 \\
\hline 64 & $\begin{array}{l}\text { 1-(Methylamino)- } \\
\text { anthraquinone }\end{array}$ & $\mathrm{C}_{15} \mathrm{H}_{11} \mathrm{NO}_{2}$ & {$[\mathrm{M}+\mathrm{H}]^{+}$} & 238.0863 & 0.6 & $\begin{array}{l}223.0628 \\
165.0699\end{array}$ & 7.8 & 0.2 & 0.5 \\
\hline 65 & 4-aminoazobenzene & $\mathrm{C}_{12} \mathrm{H}_{11} \mathrm{~N}_{3}$ & {$[\mathrm{M}+\mathrm{H}]^{+}$} & 198.1026 & 0.3 & $\begin{array}{l}95.0478 \\
105.0447\end{array}$ & 6.9 & 0.1 & 0.1 \\
\hline 66 & Lithol rubine BK & $\mathrm{C}_{18} \mathrm{H}_{12} \mathrm{CaN}_{2} \mathrm{O}_{6} \mathrm{~S}$ & {$[\mathrm{M}+\mathrm{H}-\mathrm{Ca}]^{-}$} & 385.05 & 0.8 & $\begin{array}{l}187.0401 \\
143.0502\end{array}$ & 5.55 & 10 & 10 \\
\hline 67 & Sudan red B & $\mathrm{C}_{24} \mathrm{H}_{20} \mathrm{~N}_{4} \mathrm{O}$ & {$[\mathrm{M}+\mathrm{H}]^{+}$} & 381.171 & 1.7 & $\begin{array}{l}224.1196 \\
106.0651\end{array}$ & 11.29 & 1 & 1 \\
\hline 68 & Solvent yellow 21 & $\mathrm{C}_{34} \mathrm{H}_{25} \mathrm{CrN}_{8} \mathrm{O}_{6}$ & {$[\mathrm{M}-\mathrm{H}]^{-}$} & 692.123 & 1.3 & $\begin{array}{l}475.0616 \\
648.1332\end{array}$ & 6.92 & 1 & 1 \\
\hline 69 & Solvent orange 62 & $\mathrm{C}_{32} \mathrm{H}_{23} \mathrm{CrN}_{10} \mathrm{O}_{8}$ & {$[\mathrm{M}-\mathrm{H}]^{-}$} & 726.1033 & 1.5 & $\begin{array}{l}447.0146 \\
540.0361\end{array}$ & 7.96 & 1 & 1 \\
\hline 70 & Solvent red 8 & $\mathrm{C}_{32} \mathrm{H}_{26} \mathrm{CrN}_{10} \mathrm{O}_{8}$ & {$[\mathrm{M}-\mathrm{H}]^{-}$} & 729.1268 & 2.2 & $\begin{array}{l}246.9691 \\
447.0151\end{array}$ & 7.84 & 1 & 1 \\
\hline 71 & Solvent yellow 16 & $\mathrm{C}_{16} \mathrm{H}_{14} \mathrm{~N}_{4} \mathrm{O}$ & {$[\mathrm{M}-\mathrm{H}]^{-}$} & 277.1095 & 1.1 & $\begin{array}{l}117.0458 \\
172.0642\end{array}$ & 8.58 & 1 & 1 \\
\hline 72 & Fast brown RR & $\mathrm{C}_{16} \mathrm{H}_{14} \mathrm{~N}_{4}$ & {$[\mathrm{M}+\mathrm{H}]^{+}$} & 263.1291 & 0.7 & $\begin{array}{l}143.0730 \\
246.1025\end{array}$ & 7.22 & 0.02 & 0.02 \\
\hline 73 & Oil orange SS & $\mathrm{C}_{17} \mathrm{H}_{14} \mathrm{~N}_{2} \mathrm{O}$ & {$[\mathrm{M}+\mathrm{H}]^{+}$} & 263.1179 & 0.4 & $\begin{array}{l}246.1152 \\
107.0729\end{array}$ & 9.3 & 0.02 & 0.02 \\
\hline 74 & Red 2G (Azophloxine) & $\mathrm{C}_{18} \mathrm{H}_{13} \mathrm{~N}_{3} \mathrm{Na}_{2} \mathrm{O}_{8} \mathrm{~S}_{2}$ & {$[\mathrm{M}+\mathrm{H}-2 \mathrm{Na}]^{-}$} & 464.0228 & 1.1 & $\begin{array}{l}358.9775 \\
263.9972\end{array}$ & 4.06 & 2 & 2 \\
\hline 75 & $\mathrm{Cu}(\mathrm{II})$ Chlorin e4 & $\mathrm{C}_{33} \mathrm{H}_{32} \mathrm{CuN}_{4} \mathrm{Na}_{2} \mathrm{O}_{4}$ & {$[\mathrm{M}+\mathrm{H}-2 \mathrm{Na}]^{-}$} & 612.1803 & 2.3 & $\begin{array}{l}481.1459 \\
553.1670\end{array}$ & 9.19 & 10 & 20 \\
\hline 76 & $\mathrm{Cu}(\mathrm{II})$ Chlorin e6 & $\mathrm{C}_{34} \mathrm{H}_{31} \mathrm{CuN}_{4} \mathrm{Na}_{3} \mathrm{O}_{6}$ & {$[\mathrm{M}+2 \mathrm{H}-2 \mathrm{Na}]^{-}$} & 656.1702 & 3.1 & $\begin{array}{l}522.1850 \\
507.1615\end{array}$ & 10.39 & 10 & 20 \\
\hline 77 & 4-amino carminic acid & $\mathrm{C}_{22} \mathrm{H}_{21} \mathrm{NO}_{12}$ & {$[\mathrm{M}-\mathrm{H}]^{-}$} & 490.0991 & 3.7 & $\begin{array}{l}356.0776 \\
326.0670\end{array}$ & 4.12 & 0.5 & 0.5 \\
\hline 78 & Disperse orange 37 & $\mathrm{C}_{17} \mathrm{H}_{15} \mathrm{Cl}_{2} \mathrm{~N}_{5} \mathrm{O}_{2}$ & {$[\mathrm{M}+\mathrm{H}]^{+}$} & 392.0676 & 4.3 & $\begin{array}{l}351.0410 \\
165.0784\end{array}$ & 8.2 & 0.1 & 0.1 \\
\hline 79 & Guinea green B & $\mathrm{C}_{37} \mathrm{H}_{35} \mathrm{~N}_{2} \mathrm{NaO}_{6} \mathrm{~S}_{2}$ & {$[\mathrm{M}-\mathrm{Na}]^{-}$} & 667.1942 & 4.5 & $\begin{array}{l}497.1893 \\
587.2363\end{array}$ & 5.49 & 10 & 10 \\
\hline 80 & Saccharin & $\mathrm{C}_{7} \mathrm{H}_{5} \mathrm{NO}_{3} \mathrm{~S}$ & {$[\mathrm{M}-\mathrm{H}]^{-}$} & 181.9917 & 4.2 & $\begin{array}{l}105.9598 \\
92.9186\end{array}$ & 2.83 & 0.02 & 0.02 \\
\hline 81 & Cyclamate & $\mathrm{C}_{6} \mathrm{H}_{13} \mathrm{NO}_{3} \mathrm{~S}$ & {$[\mathrm{M}-\mathrm{H}]^{-}$} & 178.0543 & 4.1 & $\begin{array}{l}79.9568 \\
94.9955\end{array}$ & 3.02 & 0.1 & 0.1 \\
\hline 82 & Aspartame & $\mathrm{C}_{14} \mathrm{H}_{18} \mathrm{~N}_{2} \mathrm{O}_{5}$ & {$[\mathrm{M}+\mathrm{H}]^{+}$} & 295.1288 & 2.3 & $\begin{array}{l}120.0813 \\
103.0547\end{array}$ & 4.03 & 0.1 & 0.1 \\
\hline 83 & Stevioside & $\mathrm{C}_{38} \mathrm{H}_{60} \mathrm{O}_{18}$ & {$[\mathrm{M}-\mathrm{H}]^{-}$} & 803.3706 & 1.6 & $\begin{array}{l}641.3231 \\
317.2116\end{array}$ & 5.58 & 0.1 & 0.1 \\
\hline 84 & Acesulfame potassium & $\mathrm{C}_{4} \mathrm{H}_{5} \mathrm{NO}_{4} \mathrm{~S}$ & {$[\mathrm{M}-\mathrm{H}]^{-}$} & 161.9866 & 5.2 & $\begin{array}{l}82.0292 \\
77.9649\end{array}$ & 1.36 & 0.02 & 0.02 \\
\hline 85 & Dulcin & $\mathrm{C}_{9} \mathrm{H}_{12} \mathrm{~N}_{2} \mathrm{O}_{2}$ & {$[\mathrm{M}+\mathrm{H}]^{+}$} & 181.0971 & 4.3 & $\begin{array}{l}108.0449 \\
65.0391\end{array}$ & 4.5 & 0.02 & 0.02 \\
\hline 86 & $\begin{array}{l}\text { Neohesperidin } \\
\text { dihydrochalcone }\end{array}$ & $\mathrm{C}_{28} \mathrm{H}_{36} \mathrm{O}_{15}$ & {$[\mathrm{M}+\mathrm{H}]^{+}$} & 613.2127 & 0.9 & $\begin{array}{l}137.0602 \\
179.0708\end{array}$ & 4.89 & 1 & 1 \\
\hline 87 & Glycyrrhizin & $\mathrm{C}_{42} \mathrm{H}_{61} \mathrm{O}_{16}$ & {$[\mathrm{M}-\mathrm{H}]^{-}$} & 821.3959 & 0.3 & $\begin{array}{l}351.0563 \\
113.0239\end{array}$ & 5.62 & 0.1 & 0.1 \\
\hline 88 & Neotame & $\mathrm{C}_{20} \mathrm{H}_{30} \mathrm{~N}_{2} \mathrm{O}_{5}$ & {$[\mathrm{M}+\mathrm{H}]^{+}$} & 379.2227 & 2.9 & $\begin{array}{l}172.1337 \\
259.181\end{array}$ & 5.52 & 0.1 & 0.1 \\
\hline 89 & Alitame & $\mathrm{C}_{14} \mathrm{H}_{25} \mathrm{~N}_{3} \mathrm{O}_{4} \mathrm{~S}$ & {$[\mathrm{M}+\mathrm{H}]^{+}$} & 332.1638 & 3.3 & $\begin{array}{l}129.0738 \\
159.0769\end{array}$ & 4.3 & 0.1 & 0.1 \\
\hline 90 & Rebaudioside A & $\mathrm{C}_{44} \mathrm{H}_{70} \mathrm{O}_{23}$ & {$[\mathrm{M}-\mathrm{H}]^{-}$} & 965.4235 & 2.1 & $\begin{array}{l}803.3755 \\
641.3168\end{array}$ & 5.13 & 10 & 10 \\
\hline
\end{tabular}


Table 1. (continued)

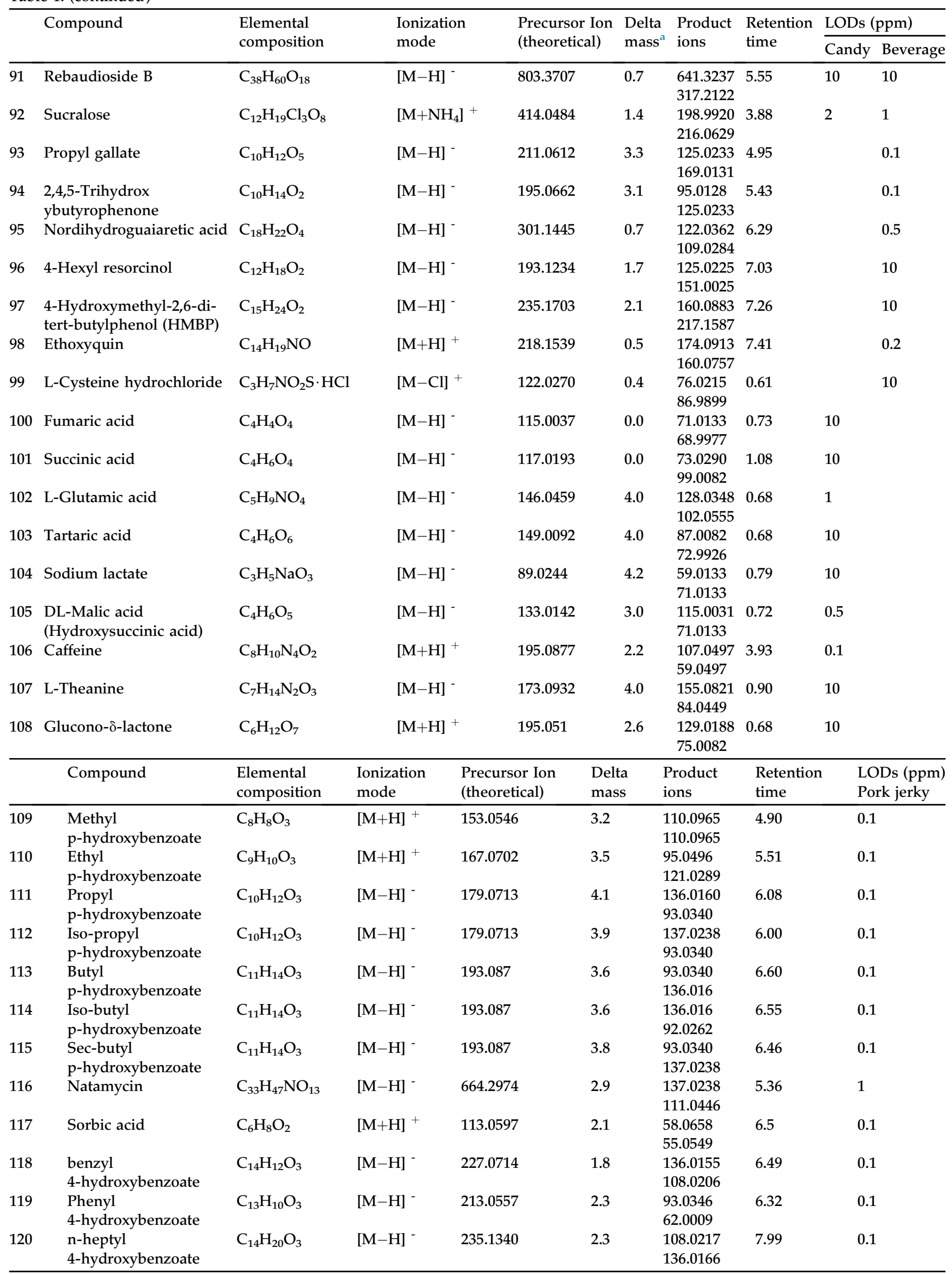

a Difference between the measured mass/charge $(\mathrm{m} / \mathrm{z})$ of procurer and the exact $\mathrm{m} / \mathrm{z}$ of that ion. Expressed as $\delta \mathrm{ppm}$.

b Compound is not soluble in water. 
the mixed precursors in every wide window. Theoretically, DIA was able to record all chemicals with the required information of precursors and product ions in a sample as long as they were able to be ionized by ESI. The identification of analytes was performed according to SANTA/11813/2017 guidelines [19]. Mass accuracy less than 5 ppm was applied for 2 fragments, and less than 10 ppm was for a precursor. Matched retention time was limited in $0.2 \mathrm{~min}$. Signal was required to have $S / N$ ratio larger than 3 or at least 5 subsequent scans presented (in some case $\mathrm{S} / \mathrm{N}$ ratio can not be calculated due to $\mathrm{S} / \mathrm{N}$ ratio = infinity). A total ion chromatogram of sample 22 as an example was shown in Fig. 2a, and the presence of allura red AC was shown in Fig. $2 b$ which data was processed by selecting a product ion $\mathrm{m} / \mathrm{z} 205.9917$ from a group of precursors in the range of $\mathrm{m} / z$ 449-476 (accurate mass of allura red AC was 451.0275). The scan spectrum of $\mathrm{RT}=4.05$ (from full scan chromatogram, Fig. 2a) was showed in Fig. 2c. There were various compounds co-elute and each one can be a precursor. In DIA, isolation window 27 amu was applied for $m / z 100$ to 500 in every 25 amu increment. Therefore, the precursor ion of allura red AC was selected along with other ions presented in the range of $\mathrm{m} / \mathrm{z} 449$ to 476 (Fig. 2d). Multiple precursors were fragmented together, and the mixed product ions were shown in Fig. 2e. Two characteristic ions 79.95695 and 205.99075 indicated the positive identification of allura red AC.

\subsection{LODs for the screening method}

Colorants were tested in candy and carbonated sparkling water for LODs evaluation. The obtained LODs ranged between 0.02 and 20 part per million (ppm), and mostly $0.5 \mathrm{ppm}$ in Table 1 . Antioxidants and flavoring agents observed LODs at 1-10 ppm range. Preservatives were tested in pork jerky
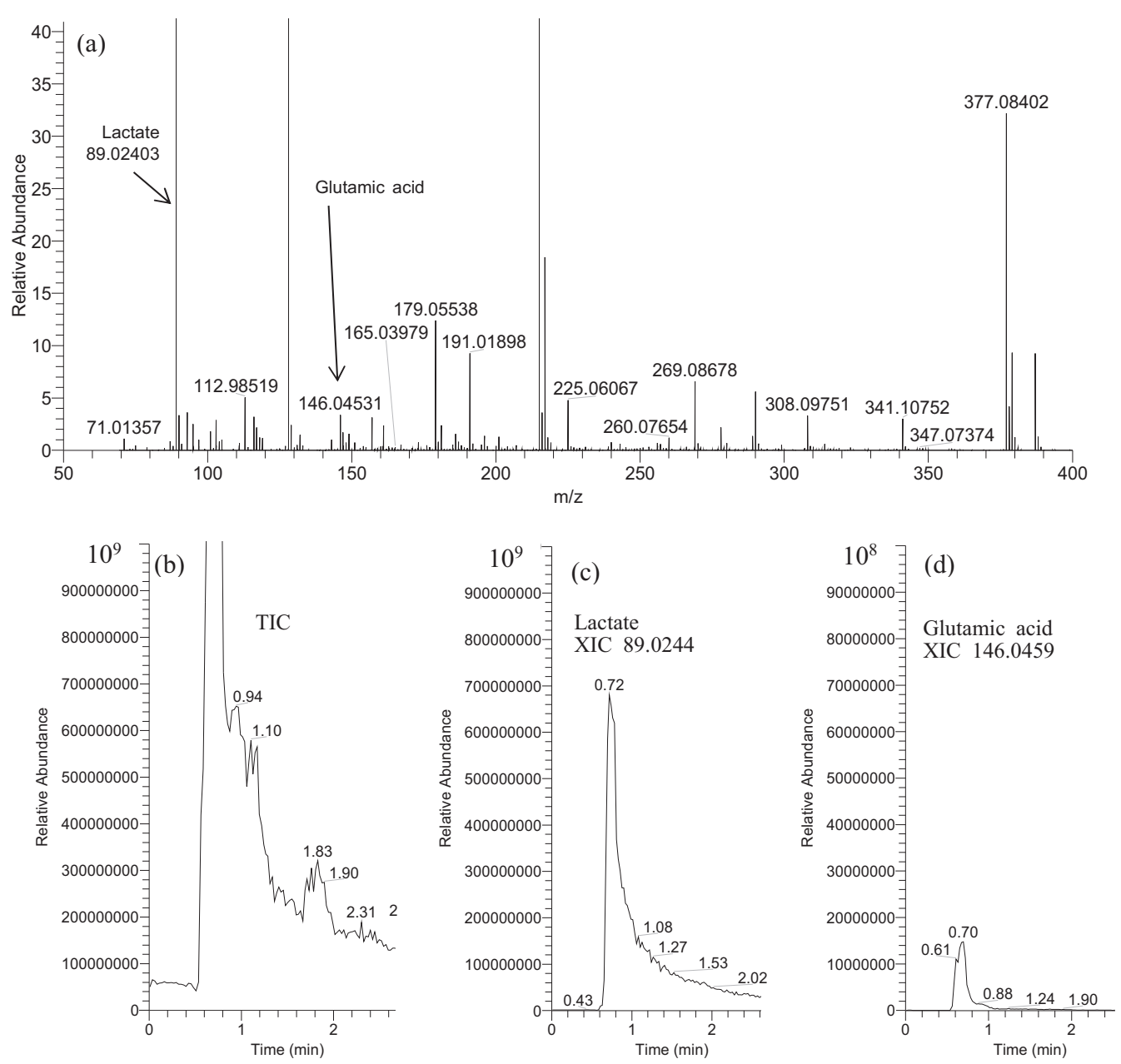

Fig. 1. Chromatograms and MS spectrum of a soy sauce sample. a) ESI negative spectrum of $R T=0.7$ min; b) chromatogram of RT around dead time $(0.7 \mathrm{~min}) ; \mathrm{c})$ chromatogram of lactate utilizing XIC 89.0244; d) chromatogram of glutamic acid utilizing XIC 146.0459. 

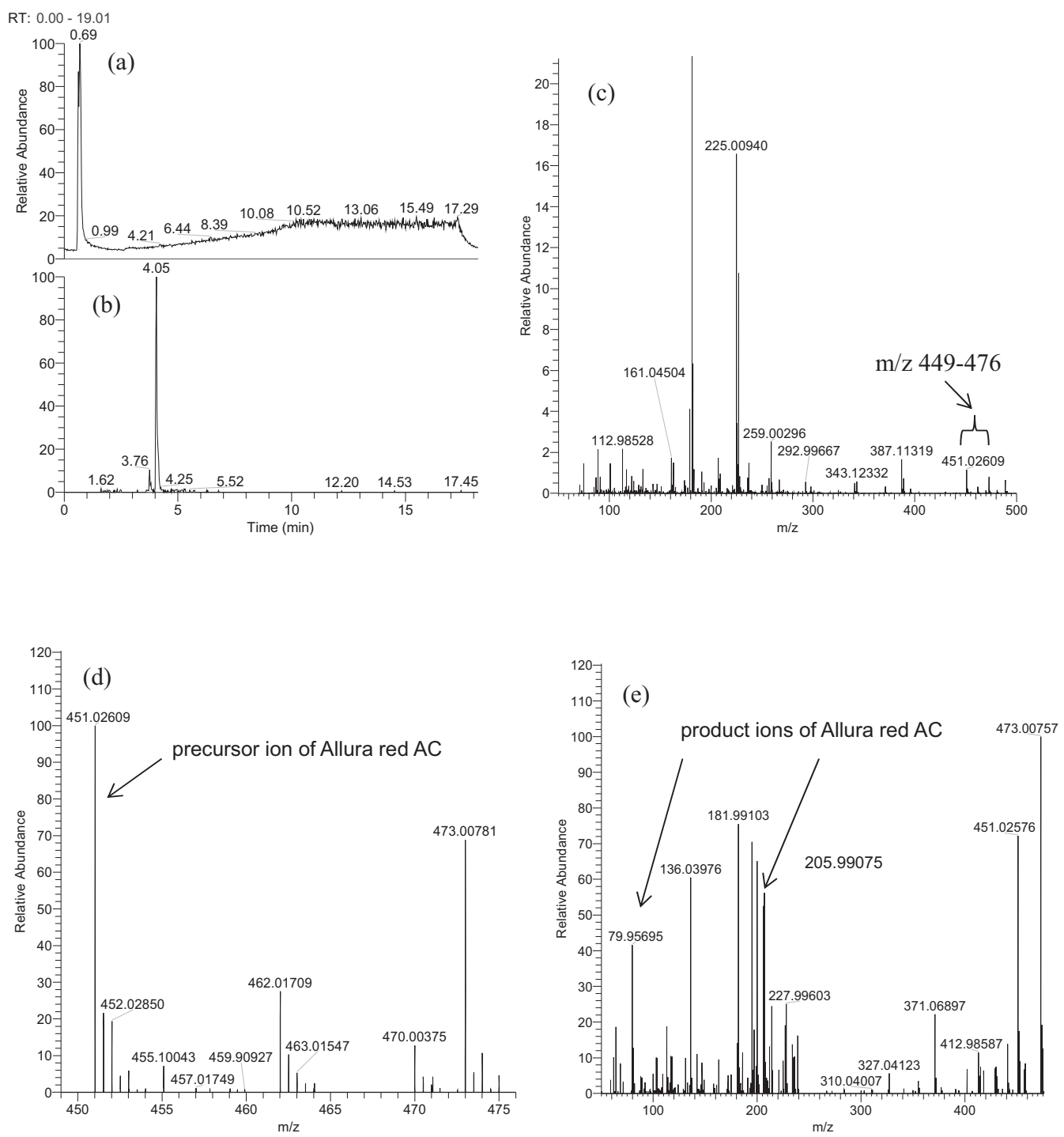

Fig. 2. Chromatograms and MS spectra of a mints candy sample. a) total ion chromatogram; b) chromatogram of product ion $\mathrm{m} / \mathrm{z} 205.99075$ from precursors in the range of $\mathrm{m} / \mathrm{z} 449-476 ; c)$ ESI full scan $M S$ spectrum of $R T=4.05 ; d)$ multiple precursors in a wide window selected in the range of $\mathrm{m} / \mathrm{z} 449-476 ;$ e) spectrum of mixed product ions for multiple precursors.

matrix and obtained LODs between 0.1 and 1 ppm. In the comparison to other multi-residue studies for additives by targeted acquisition method [9], this nontargeted data acquisition provided 10 to 100 -fold higher LODs. Conventional targeted method optimized ionization and fragmentation parameters for each analyte such as cone voltage for precursors and collision energy for product ions. However, nontargeted method was not able to optimize MS parameter for "non target", instead a basic or typical MS parameter was applied for a broad compound screening or called high throughput screening. Colorants such as rhodamine B observed similar LOD to a targeted MRM method. Cu(II) Chlorin e4 and $\mathrm{Cu}(\mathrm{II})$ Chlorin e6 observed LODs as $20 \mathrm{ppm}$ in this study, but a targeted MRM method reported
$20 \mathrm{ppb}$ as the LODs and $50 \mathrm{ppb}$ as the LOQs [20]. Therefore, nontargeted method utilizing DIA is able to screen a broad list of analyte, and also suitable for food additive screening in which the detection level is around ppm level. However, for trace level analysis such as pesticide or veterinary drug residues, DIA screening may not applicable in such low detection level (10-100 ppb), and traditional optimized targeted MRM method with sample preparation for limited analytes is still suggested.

\subsection{Surveillance results of samples form commercial market}

Thirty three foods were tested utilizing the DIA method. The detected additives and labeled 
Table 2. Surveillance results of food sample form local market.

\begin{tabular}{|c|c|c|c|}
\hline No. & Product & Product form & Labeled additives \\
\hline 1 & Sparkling water (lemon) & Taiwan & $\begin{array}{l}\text { Citric acid, Flavoring (Gum arabic, } \\
\text { Sucrose acetoisobutyrate, Medium- } \\
\text { chain triglyceride), Sodium citrate, } \\
\text { Vitamin C, Carithamine, Vitamin B6 }\end{array}$ \\
\hline 2 & Orange soda & Taiwan & $\begin{array}{l}\text { Citric acid, Gum arabic, Flavoring, } \\
\text { Glycerol, Vitamin C, Vitamin E, Stevia, } \\
\text { Medium-chain triglyceride, Glycerol } \\
\text { ester of wood rosin, Carotene, sodium } \\
\text { carbonate }\end{array}$ \\
\hline 3 & Sparkling water (grape) & Taiwan & $\begin{array}{l}\text { Flavoring, Citric acid, Vitamin } C \text {, } \\
\text { Tartaric acid, Sodium citrate, Allura red } \\
\text { AC, Brilliant blue FCF }\end{array}$ \\
\hline
\end{tabular}

$4 \quad$ Apple soda

Taiwan

5

Berry vinegar drink

Taiwan

6

Apple soda

Taiwan

7

Apple vinegar drink

Taiwan

8

Soft drink

9

Sparkling grape drink

Taiwan

Zero soda

Taiwan

Mints (grape)

Taiwan

Taiwan
Flavoring, DL-Malic acid, Caramel colors, Stevia

Citric acid, flavoring, Sodium erythorbate, Sodium metaphosphate, Sodium polyphosphate, Anthocyanin, Sodium pyrophoaphate

Apple flavoring, Caramel colors, Citric acid, Natural apple flavoring

Citric acid, flavoring, Anthocyanin, Sodium erythorbate, Sodium citrate, Sodium metaphosphate, Sodium polyphosphate, Sodium pyrophoaphate Citric acid, Sodium citrate, Vitamin C, Flavoring, Acesulfame potassium, Aspartame, Beta-carotene Grape falvoring, Citric acid, Grape skin pigment

Flavoring, Citric acid, Erythritol, Vitamin C, DL-Malic acid, Monosodium L-aspartate, Sucralose, Acesulfame potassium, Vitamin B1, Vitamin B2, Vitamin B6 Sorbitol, Aspartame, Acesulfame potassium, Malic acid, Grape flavoring, Magnesium stearate, Silicon dioxide, Erythrosine, Brilliant blue

Sorbitol, Aspartame, Acesulfame potassium, Malic acid, Soda flavoring, Magnesium stearate, Silicon dioxide, Brilliant blue

Sorbitol, Aspartame, Acesulfame potassium, Peach flavoring, Malic acid, Magnesium stearate, Silicon dioxide, Erythrosine

Sorbitol, Acesulfame potassium, Sucralose, Magnesium stearate, Flavoring, DL-Malic acid, Citric acid, Allura red AC

Sorbitol, Acesulfame potassium, Sucralose, Magnesium stearate, Flavoring, Lactic acid, Calcium lactate, Indigo carmine

Sorbitol, Acesulfame potassium, Sucralose, Flavoring, Magnesium stearate, Brilliant blue FCF
Detected additives

Rebaudioside A, DL-Malic acid, Fumaric acid, Glucono- $\delta$-Lacton, Sodium Lactate

Rebaudioside A, DL-Malic acid, Fumaric acid, Glucono- $\delta$-Lactone, LGlutamic acid, Sodium Lactate, Tartaric acid

Tartaric acid, Glucono- $\delta$-Lactone, Sodium Lactate, DL-Malic acid, Fumaric acid, Succinic acid, Allura red AC, Brilliant blue FCF

DL-Malic acid, Fumaric acid, Glucono$\delta$-Lactone, L-Glutamic acid, Sodium Lactate, Tartaric acid, Succinic acid Sodium Lactate, DL-Malic acid, Fumaric acid, Tartaric acid

Rebaudioside A, DL-Malic acid, Fumaric acid, Glucono- $\delta$-Lactone, Sodium Lactate DL-Malic acid, Fumaric acid, Glucono$\delta$-Lactone, Sodium Lactate, Succinic acid

Aspartame, Acesulfame potassium, Fumaric acid, Sodium Lactate

DL-Malic acid, Fumaric acid, Glucono$\delta$-Lactone, L-Glutamic acid, Sodium Lactate, Tartaric acid, Succinic acid DL-Malic acid, Acesulfame potassium, Sucralose

Tartaric acid, DL-Malic acid, Sodium Lactate, Aspartame, Acesulfame potassium, Erythrosine, Brilliant blue FCF

DL-Malic acid, Fumaric acid, Sodium Lactate, L-Glutamic acid, Aspartame, Acesulfame potassium, Brilliant blue FCF

DL-Malic acid, Sodium Lactate, Succinic acid, Aspartame, Acesulfame potassium, Erythrosine

DL-Malic acid, Acesulfame potassium, Sucralose, Allura red AC

Sodium Lactate, Acesulfame potassium、Sucralose, Indigo carmine

Aspartame, Acesulfame potassium, Sucralose, Brilliant blue FCF 
Table 2. (continued)

\begin{tabular}{|c|c|c|c|c|}
\hline No. & Product & Product form & Labeled additives & Detected additives \\
\hline 17 & Mints (lemon) & Thailand & $\begin{array}{l}\text { Isomalt, Citric acid, Lactic acid, } \\
\text { Flavoring, Sucralose, Tartrazine, } \\
\text { Brilliant blue FCF }\end{array}$ & $\begin{array}{l}\text { Sodium Lactate, Sucralose, Allura red } \\
\text { AC, Tartrazine, Brilliant blue FCF }\end{array}$ \\
\hline 18 & Mints (berries) & Thailand & $\begin{array}{l}\text { Isomalt, Flavoring, Citric acid, DL-Malic } \\
\text { acid, Sucralose, Acefulfame potassium, } \\
\text { Allura red AC, Tartrazine }\end{array}$ & $\begin{array}{l}\text { DL-Malic acid, Sucralose, Acesulfame } \\
\text { potassium, Allura red AC }\end{array}$ \\
\hline 19 & Mints (honey) & Thailand & $\begin{array}{l}\text { Isomalt, Citric acid, Flavoring, } \\
\text { Sucralose, Tartrazine }\end{array}$ & Sucralose, Tartrazine \\
\hline 20 & Mints (honey and lemon) & India & $\begin{array}{l}\text { Isomalt, Aspartame, Acefulfame } \\
\text { potassium, Flavoring, Citric acid, Beta- } \\
\text { carotene }\end{array}$ & Aspartame, Acesulfame potassium \\
\hline 21 & Mints & India & $\begin{array}{l}\text { Isomalt, Aspartame, Acefulfame } \\
\text { potassium, Flavoring, Curcumin, } \\
\text { Brilliant blue FCF }\end{array}$ & $\begin{array}{l}\text { Aspartame, Acesulfame potassium, } \\
\text { Curcumin, Brilliant blue FCF }\end{array}$ \\
\hline 22 & Mints (fruits) & Australia & $\begin{array}{l}\text { DL-Malic acid, Starch acetate, } \\
\text { Flavoring, Sodium citrate, Xanthan } \\
\text { gum, Titanium dioxide, Starch sodium } \\
\text { succinate, Palm wax, Sunset yellow } \\
\text { FCF, Allura red AC, Brilliant blue FCF }\end{array}$ & $\begin{array}{l}\text { DL-Malic acid, Fumaric acid, } \\
\text { Tartrazine, Sunset yellow FCF, Allura } \\
\text { red AC, Brilliant blue FCF }\end{array}$ \\
\hline 23 & Lollipop (apple) & Vietnam & $\begin{array}{l}\text { Lactic acid, DL-Malic acid, Citric acid, } \\
\text { Flavoring, Curcumin }\end{array}$ & $\begin{array}{l}\text { Sodium Lactate, DL-Malic acid, } \\
\text { Curcumin }\end{array}$ \\
\hline 24 & Mints (strawberry) & Vietnam & $\begin{array}{l}\text { Lactic acid, DL-Malic acid, Citric acid, } \\
\text { Flavoring, Betarubin }\end{array}$ & Sodium Lactate, DL-Malic acid \\
\hline 25 & Chewing gum (mint) & Taiwan & $\begin{array}{l}\text { Sorbitol, Maltitol, D-Xylitol, D- } \\
\text { Mannitol, Aspartame, Acesulfame } \\
\text { potassium, Sucralose, Glycerol, } \\
\text { Flavoring, Sodium carboxymethyl } \\
\text { cellulose, Palm wax, Sodium alginate, } \\
\text { BHT, Brilliant blue FCF, }\end{array}$ & $\begin{array}{l}\text { Aspartame, Acesulfame potassium, } \\
\text { Sucralose, Brilliant blue FCF }\end{array}$ \\
\hline 26 & Grape C & Taiwan & $\begin{array}{l}\text { Grape powder (oxidized starch, citric } \\
\text { acid, flavoring, grape skin pigment), } \\
\text { Vitamin C, Hydropropyl } \\
\text { methylcellulose, Citric acid, Cellulose, } \\
\text { Grape flavoring, Magnesium stearate, } \\
\text { Ferric pyrophosphate, Folic acid, } \\
\text { Vitamin B12 (sodium citrate, citric acid, } \\
\text { vitamin B12) }\end{array}$ & $\begin{array}{l}\text { Tartaric acid, L-Glutamic acid, Sodium } \\
\text { Lactate, DL-Malic acid }\end{array}$ \\
\hline 27 & Mints & Australia & $\begin{array}{l}\text { Starch acetate, Flavoring, Xanthan gum, } \\
\text { Palm way, Starch sodium succinate, } \\
\text { Titanium dioxide, Brilliant blue FCF }\end{array}$ & Fumaric acid, Succinic Acid \\
\hline 28 & Mints & Taiwan & $\begin{array}{l}\text { Sorbitol, Maltitol, Maltitol syrup, D- } \\
\text { Mannitol, Aspartame, Acesulfame } \\
\text { potassium, Flavoring, Sodium } \\
\text { carboxymethyl cellulose, Palm wax, } \\
\text { Sodium alginate, BHT, BHA, Brilliant } \\
\text { blue FCF, }\end{array}$ & $\begin{array}{l}\text { Aspartame, Acesulfame potassium, } \\
\text { Brilliant blue FCF }\end{array}$ \\
\hline 29 & Soy sauce & Taiwan & Glycyrrhizin & $\begin{array}{l}\text { Tartaric Acid, Glucono- } \delta \text {-Lactone, DL- } \\
\text { Malic Acid, Fumaric Acid, L-Glutamic } \\
\text { Acid, Sodium Lactate, Succinic Acid, } \\
\text { Glycyrrhizic acid }\end{array}$ \\
\hline 30 & Soy sauce & Taiwan & $\begin{array}{l}\text { Acetylated distarch adipate, Corn } \\
\text { syrup, Glycin, Sodium succinate }\end{array}$ & $\begin{array}{l}\text { L-Glutamic Acid, Fumaric Acid, DL- } \\
\text { Malic Acid, Sodium Lactate, Succinic } \\
\text { Acid }\end{array}$ \\
\hline 31 & Soy sauce & Taiwan & Maltitol syrup & $\begin{array}{l}\text { L-Glutamic Acid, Fumaric Acid, DL- } \\
\text { Malic Acid, Sodium Lactate, Succinic } \\
\text { Acid }\end{array}$ \\
\hline 32 & Soy sauce & Taiwan & None & $\begin{array}{l}\text { Glucono- } \delta \text {-Lactone, L-Glutamic Acid, } \\
\text { Sodium Lactate, DL-Malic Acid, } \\
\text { Succinic Acid }\end{array}$ \\
\hline 33 & Soft dring & Taiwan & $\begin{array}{l}\text { Flavoring, Citric acid, Vitamin C, DL- } \\
\text { Malic acid, L-Aspartic acid, Vitamin B2, } \\
\text { Vitamin B6, Vitamin B1 }\end{array}$ & DL-Malic acid \\
\hline
\end{tabular}


additives were listed in Table 2. Sample 1, 6 were sparkling water and soda found under declared rebaudioside $\mathrm{A}$ as sweetener, which might come from the flavoring agent in their ingredients. For other drinks and candies, fumaric acid, glucono- $\delta$ lacton, sodium lactate, DL-malic acid, L-glutamic acid, tartaric acid and succinic acid were the most often flavorings under declared, which might come from natural extracts, flavoring agent mixture or artificial additives in their ingredients. Some colorants such as tartrazine (in sample 18, red candy in appearance) and Brilliant blue FCF (in sample 27, white mints in appearance) were labeled in product package, but not detected. Artificial sweeteners such acefulfame potassium, sucralose and aspartame which belong to regular sweetener testing list were all clear labeled in the package and detected. This surveillance results showed that all tested samples were all complied with regulation, but there is still room for manufacture to improve clearly declaration of product information to consumer.

\section{Conclusions}

This study established a nontargeted DIA analytical method and a detection database list of 120 food additives for rapid screening of food products. The database included 79 colorants, 13 sweeteners, 12 preservatives and 7 antioxidants, enclosed the chromatographic retention time, accurate mass of molecular ion, accurate mass of two product ions, LODs in matrix (candy, beverage or jerky). HRMS enabled secondary separation. Some high polar flavoring agent, such as lactate, L-theanine and glutamic acid were able to be separated and determined by HRMS in coelutant. However, due to the normal sample preparation and the neglect of matrix effects, DIA offered higher LODs for most compounds compared to traditional targeted MRM method. Mass spectrometer worked in full scan and wide window fragmentations that recorded all components in theoretical enabled retrospective analysis for unknowns and increasable target lists. A survey of commercial products of beverages, candies and sauces showed this method can efficiently screen food for illegal additives or false labeling. The results revealed sweeteners and flavoring agents were the most under declared additives. For example, no-calorie sweetener rebaudioside A, about 150-400 times sweeter than sugar, was found in some soda. The surveillance results showed the current labeling is good, but still needed for improvement. The detection database will continually be expand to increase the detection range of food additives for this rapid screening method.

\section{Conflict of interest}

There is no potential conflict of interest to declare.

\section{References}

[1] Lehto S, Buchweitz M, Klimm A, Straburger R, Bechtold C, Ulberth F. Comparison of food colour regulations in the EU and the US: a review of current provisions. Food Addit Contam part A 2017;34:335-55.

[2] He L, Su Y, Fang B, Shen X, Zeng Z, Liu Y. Determination of Sudan dye residues in eggs by liquid chromatography and gas chromatography-mass spectrometry. Anal Chim Acta 2007;594:139-46.

[3] Fang M, Tsai CF, Kuo CH, Cheng HF. Detection by coupled LC-photodiode array detection and high-resolution Orbitrap MS of dimethyl and diethyl yellow dyes used illegally in processed soymilk curd. Food Addit Contam 2015;32:1730-6.

[4] Błaszczyk A, Augustyniak A, Ethoxyquin Skolimowski J. An antioxidant used in animal feed. Int J Food Sci 2013;2013: $1-12$.

[5] Sagdic O, Ozturk I, Ozkan G, Yetim H, Ekici L, Yilmaz MT. RP-HPLC-DAD analysis of phenolic compounds in pomace extracts from five grape cultivars: evaluation of their antioxidant, antiradical and antifungal activities in orange and apple juices. Food Chem 2011;126:1749-58.

[6] Nevado JJB, Robledo VR, Callado CS. Monitoring the enrichment of virgin olive oil with natural antioxidants by using a new capillary electrophoresis method. Food Chem 2013;133:497-504.

[7] Xue Y, Chen N, Luo C, Wang X, Sun C. Simultaneous determination of seven preservatives in cosmetics by dispersive liquid-liquid microextraction coupled with high performance capillary electrophoresis. Anal Methods 2013;5: 2391-7.

[8] Boyce MC. Simultaneous determination of antioxidants, preservatives and sweeteners permitted as additives in food by mixed micellar electrokinetic chromatography. J Chromatogr A 1999;874:369-75.

[9] Lee HM, Yang J, Lee H, Hwang IM, Hwang YS, You S. Simultaneous determination of preservatives, artificial sweeteners, and synthetic dyes in kimchi by ultra-performance liquid chromatography electrospray ionization tandem mass spectrometry (UPLC-ESI-MS/MS). Anal Lett 2019; $52: 2472-83$

[10] Tighrine A, Amir Y, Alfaro P, Mamou M, Nerin C. Simultaneous extraction and analysis of preservatives and artificial sweeteners in juices by salting out liquid-liquid extraction method prior to ultra-high performance liquid chromatography. Food Chem 2019;277:586-94.

[11] Hamelin E, Bragg W, Shaner RL, Swaim LL, Johnson RC. Comparision of high resolution and tandem mass spectrometry for the analysis of nerve agent metabolites in urine. Rapid Commun Mass Spectrom 2013;27:1697-704.

[12] Gottfried S, Herebian D. Determination of mycotoxins in food matrices using LC-MS/MS compared with high-resolution Orbitrap MS technology. Curr Anal Chem 2013;9: 99-107.

[13] Jia W, Chu X, Ling Y, Huang J, Lin Y, Chang J. Simultaneous determination of dyes in wines by HPLC coupled to quadrupole orbitrap mass spectrometry. J Separ Sci 2014;37: 782-91.

[14] Jia W, Ling Y, Lin Y, Chang J, Chu X. Analysis of additives in dairy products by liquid chromatography coupled to quadrupole-orbitrap mass spectrometry. J Chromatogr A 2014 1336:67-75. 
[15] Kaufmann A. The current role of high-resolution mass spectrometry in food analysis. Anal Bioanal Chem 2011;403: $1233-49$.

[16] Stachniuk A. LC-MS/MS determination of pesticide residues in fruits and vegetables. In: Ramawat $K$, editor. Bioactive molecules in food. Cham: Springer; 2019. https://doi.org/ 10.1007/978-3-319-78030-6_82.

[17] Rubert J, Zachariasova M, Jajslova J. Advances in high-resolution mass spectrometry based on metabolomics studies for food - a review. Food Addit Contam 2015;32:1685-708.

[18] Wang J, Chow W, Wong JW, Leung D, Chang J, Li M. Nontarget data acquisition for target analysis (nDATA) of 845 pesticide residues in fruits and vegetables using UHPLC/ESI Q-Orbitrap. Anal Bioanal Chem 2019;411:1421-31.

[19] European Union. Guidance document on analytical quality control and method validation procedures for pesticides residues analysis in food and feed. SANTE/11813/2017, https://ec.europa.eu/food/sites/food/files/plant/docs/ pesticides_mrl_guidelines_wrkdoc_2017-11813.pdf. [Accessed 17 March 2021].

[20] Fang M, Tsai CF, Wu GY, Tseng SH, Cheng HF, Kuo CH, et al. Identification and quantification of $\mathrm{Cu}$-chlorophyll adulteration of edible oils. Food Addit Contam Part B Surveill 2015;8:157-62. 